\title{
Liberalisierung des deutschen Postsektors - Auswirkungen auf Beschäftigung und Tarifpolitik
}

\author{
Torsten Brandt \\ Kathrin Drews \\ Thorsten Schulten
}

Wenige Monate vor der geplanten vollständigen Liberalisierung des deutschen Briefmarktes zum 1. Januar 2008 rücken deren absehbare arbeits- und beschäftigungspolitische Folgen immer mehr in die öffentliche Debatte. Angesichts eines stagnierenden Briefmarktes droht ein umfangreicher Verdrängungswettbewerb, der vor allem auf Kosten der Beschäftigten ausgetragen wird. Schon heute basiert das Wettbewerbsmodell der meisten neuen Postunternehmen darauf, dass prekäre Beschäftigungsverhältnisse genutzt und Niedrig- oder Armutslöhne gezahlt werden. Umso wichtiger ist es, über Ansätze für eine (tarif-)politische Re-Regulierung des Postsektors nachzudenken. ${ }^{1}$

\section{Die Liberalisierung des deutschen Postsektors}

Die Auseinandersetzung um die Liberalisierung des deutschen Postsektors reicht bis in die 1980er Jahre zurück. ${ }^{2}$ Bereits 1985 wurde von der konservativ-liberalen Bundesregierung eine „Regierungskommission Fernmeldewesen " eingesetzt, deren Aufgabe darin bestand, Vorschläge zur Neuordnung des deutschen Post- und Telekommunikationssektors zu unterbreiten. Nach dem Postgesetz von 1969 wurde dem damaligen Staatsunternehmen „Deutsche Bundespost" das exklusive Recht zur Beförderung von Briefen eingeräumt. Der eigentliche Startschuss für die Liberalisierung war im Jahre 1989 die sogenannte Postreform $I$, in deren Folge es zu einer weitgehenden Restrukturierung der Deutschen Bundespost in drei separate Unternehmenseinheiten für Postdienste, Postbank und Telekommunikation kam. Die Verabschiedung der Postreform I erfolgte gegen den Widerstand der Deutschen Postgewerkschaft (DPG) und der damals oppositionellen SPD, die sich gegen die hierdurch vorbereitete spätere Privatisierung der Deutschen Post aussprachen.

Mit der Postreform II fand 1995 die Umwandlung der drei Unternehmen in eigenständige Aktiengesellschaften statt, womit aus der Postsparte der ehemaligen Deutschen Bundespost nunmehr die Deutsche Post AG (DPAG) wurde. Zuvor war allerdings eine Änderung des Grundgesetzes (GG) notwendig, da die Deutsche Bundespost in der ursprünglichen Version von Ar- tikel 87 GG als Teil der bundeseigenen Verwaltung definiert wurde. Während die Deutsche Postgewerkschaft sich bis zuletzt gegen eine Änderung des Grundgesetzes aussprach, vollzog die SPD im Hinblick auf die Privatisierung der Post einen grundlegenden Positionswechsel und ermöglichte damit die für eine Verfassungsänderung notwendige Zweidrittelmehrheit im Bundestag (Wehner 2005, S. 10f.). Mit dem neu geschaffenen Artikel 87f. GG werden die Postdienste nun als privatwirtschaftliche Tätigkeiten angesehen, die prinzipiell sowohl durch die Deutsche Post als auch durch andere private Anbieter erbracht werden können. Außerdem wurde mit dem ebenfalls neu hinzugefügten Artikel 143b GG die Umwandlung der Deutschen Bundespost in Unternehmen mit privater Rechtsform beschlossen. Allerdings behält der Bund nach Artikel 87f. GG die Verantwortung für die Gewährleistung „flächendeckend angemessener und ausreichender Dienstleistungen" und sichert sich damit auf dem Postmarkt weitreichende Regulierungskompetenzen.

Seinen vorläufigen Höhepunkt erreichte der Prozess der Liberalisierung 1998 mit der Postreform III, die mit der Verabschiedung eines neuen Postgesetzes eine sukzessive Einschränkung der Exklusivlizenz der Deutschen Post AG und die schrittweise Öffnung des Briefmarktes für private Anbieter vorsah. Hierbei wurde die Gewichtsgrenze für Briefe, die unter das Briefmonopol fallen, schrittweise abgesenkt. Ursprünglich sollten bereits 2002 alle Wettbewerbsbeschränkungen aufgehoben werden. Nachdem sich in den meisten anderen europäischen Staaten die Liberalisierung der Postmärkte jedoch deutlich langsamer vollzog, wurde das endgültige Ende des Briefmonopols auf den 1. Januar 2008 verschoben. Bis dahin wird der Deutschen Post AG eine Exklusivlizenz für Briefe bis 50 Gramm eingeräumt.

Der Börsengang im November 2000 markiert schließlich den Beginn der „materiellen" Privatisierung der Deutschen Post. Seit 2005 befindet sich der nun unter dem Namen „Deutsche Post World Net“ (DPWN) firmierende Konzern mehrheitlich in privater Hand, wobei die Aktienanteile vorwiegend von ausländischen institutionellen Investoren gehalten werden.

\footnotetext{
1 Der Beitrag entstand im Rahmen eines von der Europäischen Kommission geförderten Forschungsprojektes „Privatisation of Public Services and the Impact on Quality Employment and Productivity" (PIQUE) www.pique.at.

2 Zur Geschichte der Liberalisierung des deutschen Postsektors vgl. Cox (1999), Drews (2006), Lotz (2007), sowie aus gewerkschaftlicher Perspektive Wehner (2005)
}

Torsten Brandt, Wissenschaftler im WSI in der Hans-Böckler-Stiftung (Projekt PIQUE). Arbeitsschwerpunkte: Privatisierung öffentlicher Dienstleistungen, Arbeitsmarktpolitik, Industrielle Beziehungen. e-mail: Torsten-Brandt@boeckler.de Kathrin Drews, Studentin der Politikwissenschaft an der Philipps-Universität Marburg. e-mail:drewsk@students.uni-marburg.de Thorsten Schulten, Dr., Wissenschaftler im WSI in der Hans-Böckler-Stiftung. Arbeitsschwerpunkte: Arbeits- und Tarifpolitik in Europa.

e-mail: Thorsten-Schulten@boeckler.de 
Der Bund hält über die Kreditanstalt für Wiederaufbau (KfW) noch etwa ein Drittel der Aktienanteile an der Deutschen Post (DPWN 2007a, S. 53).

\section{Der Einfluss der Europäischen Union}

Seit Anfang der 1990er Jahre wird in Deutschland - wie in den meisten europäischen Ländern - die Entwicklung des Postsektors in hohem Maße durch die Politik der Europäischen Union (EU) beeinflusst (Haas et al. 2004; Lippert 2005). Mit der Vorlage eines Grünbuches über die Entwicklung des Binnenmarktes für Postdienste im Jahr 1992 und der Verabschiedung der ersten Postrichtlinie im Jahr 1997 hat die EU einen politischen Fahrplan für eine europaweite Liberalisierung des Postmarktes vorgegeben. ${ }^{3}$ Mit ihrer Politik der forcierten Marktöffnung hat die EU auch die deutschen Postreformen II und III maßgeblich beeinflusst. Das Postgesetz von 1998 stellt demnach eine direkte Umsetzung der im Vorjahr verabschiedeten ersten europäischen Postrichtlinie in nationales Recht dar.

Die Privatisierung der ehemals staatlichen Post-Unternehmen kann zwar nicht unmittelbar auf europäisches Recht zurückgeführt werden, da EU-Richtlinien eigentumsrechtlich neutral bleiben und nicht explizit eine Privatisierung öffentlicher Unternehmen fordern. Durch die innerhalb der EU vorherrschende Wettbewerbsphilosophie werden die Prinzipien des Europäischen Binnenmarktes jedoch auf immer mehr gesellschaftliche Bereiche ausgedehnt. Hierdurch wird deren privatwirtschaftliche Organisation gefördert. Dem entspricht die in Deutschland vollzogene Deklaration der Postdienste als „privatwirtschaftliche Aufgabe" (Cox 1999, S. 85). Hinzu kommt, dass mit den restriktiven fiskalpolitischen Vorgaben des EU-Stabilitätspaktes sowie aufgrund der durch den europäischen Steuersenkungswettbewerb induzierten Finanznot öffentlicher Haushalte die Möglichkeiten öffentlichen Wirtschaftens systematisch begrenzt werden. Bei einer Gesamtbetrachtung der EUWirtschaftspolitik erscheinen demnach Liberalisierung und Privatisierung als "zwei Seiten derselben Medaille“ (Raza et al. 2004, S. 30).
Die Liberalisierung des deutschen Postsektors vollzog sich im Großen und Ganzen im Einklang mit den von der Europäischen Kommission vorgesehenen $\mathrm{Li}$ beralisierungsschritten. In einigen Fällen ging Deutschland sogar über die Vorgaben der EU hinaus und hat das für den Wettbewerb geöffnete Segment des Briefmarktes deutlich schneller ausgedehnt. Innerhalb Europas ist die Wettbewerbsintensität auf dem deutschen Briefmarkt heute de facto mit am größten (Input Consulting 2006a; Brandt 2007). Bislang haben lediglich Schweden, Finnland und Großbritannien eine vollständige Liberalisierung ihrer nationalen Briefmärkte vollzogen. Deutschland und die Niederlande haben diesen Schritt für 2008 angekündigt, während ein aktueller Richtlinienentwurf der Europäischen Kommission die EU-weite Vollendung des Binnenmarktes für Postdienste für das Jahr 2009 vorschlägt (Europäische Kommission 2006).

$\mathrm{Ob}$ es tatsächlich bereits $2009 \mathrm{zu}$ einer vollständigen Liberalisierung des Postmarktes in der EU kommt, ist indes derzeit recht ungewiss. Während sich die ehemaligen staatlichen Postkonzerne aus Deutschland, Schweden, Finnland, Großbritannien und den Niederlanden für eine schnelle europaweite Liberalisierung aussprechen, haben die Incumbents ${ }^{4}$ aus Frankreich, Belgien, Italien, Spanien und anderen Ländern deutliche Bedenken angemeldet, die vor allem mit der bislang noch ungeklärten Sicherstellung und Finanzierung des Universaldienstes in einem vollständig liberalisierten Postmarkt zusammenhängen. Angesichts der derzeitigen Unsicherheit über die weitere Entwicklung auf europäischer Ebene ist auch in Deutschland wieder eine Debatte über den Zeitpunkt der vollständigen Liberalisierung entbrannt. Die Deutsche Post AG plädiert mittlerweile dafür, den geplanten Termin für die endgültige Aufhebung des Briefmonopols Anfang $2008 \mathrm{zu}$ verschieben, da sie aufgrund der ungleichen Entwicklung in Europa gegenüber den - aus nach wie vor geschützten nationalen Briefmärkten agierenden ausländischen Postkonzernen erhebliche Wettbewerbsnachteile befürchtet. Auch Teile der Bundesregierung wie die sozialdemokratischen Minister Müntefering und Steinbrück haben sich aus demselben Grund für eine Verlängerung der Exklusivlizenz ausgesprochen. Demgegenüber fordern die in Deutschland neu etablierten Postunternehmen, den bestehenden Zeit- plan zum Wegfall des Briefmonopols einzuhalten; sie werden hierbei insbesondere vom Bundeswirtschaftsministerium unterstützt.

\section{Entwicklung und Struktur des deutschen Briefmarktes}

Vor der Verabschiedung des neuen Postgesetzes im Jahr 1998 waren lediglich $3 \%$ des deutschen Briefmarktes für den Wettbewerb geöffnet, sodass es für private Konkurrenzunternehmen der Deutschen Post kaum möglich war, eine relevante Marktposition zu erobern. Seither ist der Monopolbereich der Deutschen Post sukzessive eingeschränkt worden; rund die Hälfte des deutschen Briefmarktes ist mittlerweile für den Wettbewerb geöffnet (Bundesnetzagentur 2007, S. 114). Darüber hinaus haben die Konkurrenten der Deutschen Post AG auch heute schon die Möglichkeit, innerhalb des Monopolbereiches (d.h. bei Briefen bis 50 Gramm) tätig zu werden, wenn sie sogenannte "qualitativ höherwertige Dienstleistungen“, wie z. B. die Zustellung am selben Tag oder Abholung der Post beim Absender, erbringen.

Nach Angaben der Bundesnetzagentur (2007, S. 116) hatten im Jahr 20061.470 Unternehmen eine Lizenz für die Erbringung von Postdienstleistungen, von denen jedoch nur 750 aktiv am Marktgeschehen beteiligt waren. In der Mehrzahl handelt es sich hierbei um kleine Unternehmen, die vorwiegend auf lokalen und regionalen Märkten tätig sind. Bei knapp $80 \%$ der Postunternehmen lag der Umsatz im Jahr 2006 bei weniger als einer Mio. Euro. Lediglich $3 \%$ der Unternehmen hatten einen Umsatz von mehr als 10 Mio. Euro (Abbildung 1). In den letzten Jahren lässt sich allerdings ein zunehmender Konzentrationsprozess im Postsektor feststellen, bei dem sich mit der TNT Post und der Pin Group zwei weitere Großkonzerne herausgebildet

\footnotetext{
3 Alle wichtigen Dokumente zur europäischen Liberalisierungspolitik im Postsektor finden sich auf einer hierzu von der Europäischen Kommission eingerichteten Webpage: http://ec.europa.eu/internal_market/post/index_de.htm

4 Der Ausdruck „Incumbent" bezeichnet ein bei Marktöffnung bereits auf einem Markt etabliertes Unternehmen, d.h. in der Regel den ehemaligen Monopolisten.
} 
haben, die zu den Hauptkonkurrenten der Deutschen Post gehören (Input Consulting 2006b, S. 28ff.).

Die TNT Post ist ein Gemeinschaftsunternehmen des ehemaligen staatlichen niederländischen Postkonzerns TNT und der deutschen Hermes Logistik Gruppe. Die PIN Group wurde von den großen deutschen Zeitungsverlagen Axel Springer, Georg von Holtzbrinck und der WAZ-Mediengruppe gemeinsam mit der Luxemburger Beteiligungsgesellschaft Rosalia gegründet. Beide Konzerne haben durch die Übernahme lokaler Postunternehmen und den Abschluss von Kooperationspartnerschaften innerhalb Deutschlands ein weitgehend flächendeckendes Zustellnetz aufgebaut.

Obwohl der Marktanteil der Deutschen Post AG in den letzten Jahren kontinuierlich zurückgegangen ist, lag er im Jahr 2006 immer noch bei über $90 \%$ (Abbildung 2). Betrachtet man nur das bereits liberalisierte Segment des Briefmarktes, so haben die Konkurrenten der Deutschen Post mittlerweile einen Marktanteil von knapp $17 \%$ erobern können (Bundesnetzagentur 2006, S. 24). Die Deutsche Post rechnet damit, nach der vollständigen Aufhebung des Briefmonopols bis zu $20 \%$ Marktanteile zu verlieren (Zumwinkel 2007). Um diesen Marktverlust zu kompensieren, hat sich die Deutsche Post in den letzten Jahren durch zahlreiche Akquisitionen im Ausland zu einem der führenden internationalen Logistikkonzerne entwickelt (Wehner 2005, S. $15 \mathrm{ff}$.).

Die Entwicklung des deutschen Briefmarktes zeigt insgesamt bereits seit Jahren eine stagnierende Tendenz (Bundesnetzagentur 2007, S. 115). Für die Zukunft gehen die meisten Prognosen davon aus, dass dieser Trend anhält oder dass der Briefmarkt aufgrund weiterer Substitution durch elektronische Medien sogar schrumpfen wird. Vor dem Hintergrund eines im Wesentlichen gesättigten Marktes vollzieht sich der Wettbewerb im Postsektor deshalb in erster Linie als ein über Preiskonkurrenzen ausgetragener Verdrängungswettbewerb (Input Consulting 2006b, S. 38). Für eine personalintensive Branche wie die Post mit einem hohen Personalkostenanteil ist es deshalb nahe liegend, dass die Lohn- und Arbeitskosten in den Mittelpunkt des Wettbewerbs rücken.

Abb. 1: Postunternehmen in Deutschland nach Umsatzgröße 2006 - in \% -

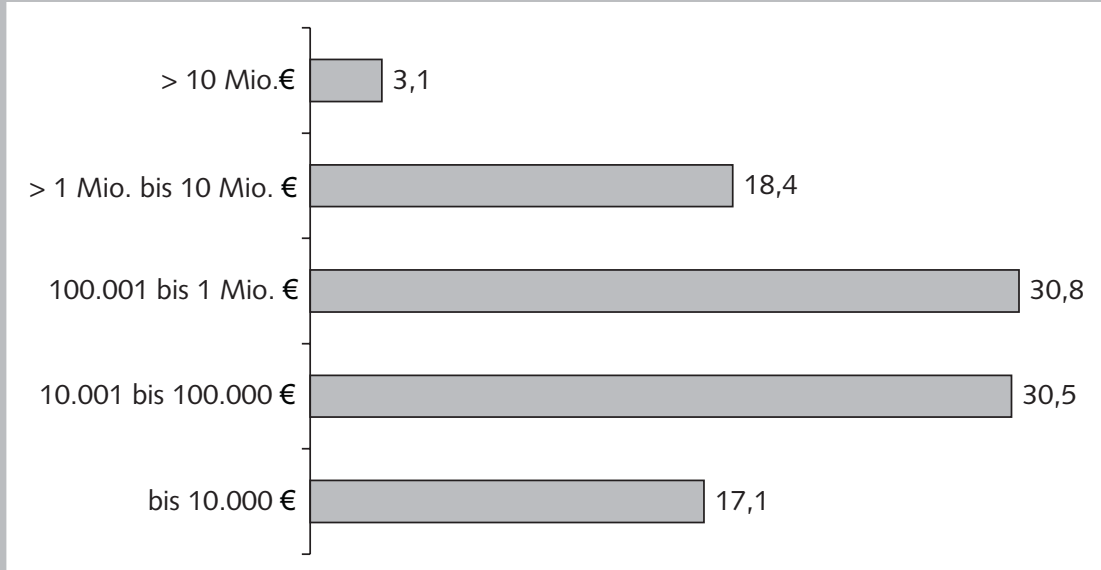

Quelle: Bundesnetzagentur (2007, S. 119); eigene Berechnungen der Autoren.

WSI

MITTEILUNGEN

\section{Auswirkungen der Liberalisierung auf Beschäftigung}

\subsection{BESCHÄFTIGUNGSENTWICKLUNG BEI DER DEUTSCHEN POST AG}

Vor Beginn der Liberalisierung Ende der 1980er Jahre hatte die damalige „Deutsche Bundespost Postdienste" noch knapp 400.000 Beschäftigte (Lotz 2007; Scheurle 2001). In den 1990er Jahren ist die Anzahl der Beschäftigten kontinuierlich gesunken und erreichte im Jahre 1998 mit etwa 260.000 ihren historischen Tiefpunkt ( $\mathrm{Ta}$ belle 1). Seither hat der Beschäftigtenstand wieder deutlich zugenommen und lag Ende 2006 bei über 520.000 Mitarbeitern. Der
Beschäftigtenzuwachs ist allerdings nahezu ausschließlich auf Firmenübernahmen im Ausland zurückzuführen. Der Anteil der in Deutschland tätigen Postbeschäftigten ist hingegen auch nach 1998 weiter zurückgegangen und dürfte mittlerweile bei deutlich unter $50 \%$ liegen. Ebenfalls kontinuierlich gesunken ist der Anteil der Beamten, der vor der Liberalisierung noch bei über $50 \%$ lag und heute mit etwas mehr als 62.000 Beschäftigten noch etwa $12 \%$ beträgt.

Einen starken Rückgang der Beschäftigung gab es insbesondere im Briefsektor, der seit den 1990er Jahren umfangreichen Rationalisierungsmaßnahmen unterzogen wurde (Scheurle 2001). Während Anfang der 1990er Jahre noch $75 \%$ der Briefe von Hand sortiert wurden, werden heute fast $90 \%$ maschinell verarbeitet. Ab 2000 sorgten außerdem organisatorische und tarifpolitische Maßnahmen für den Abbau von

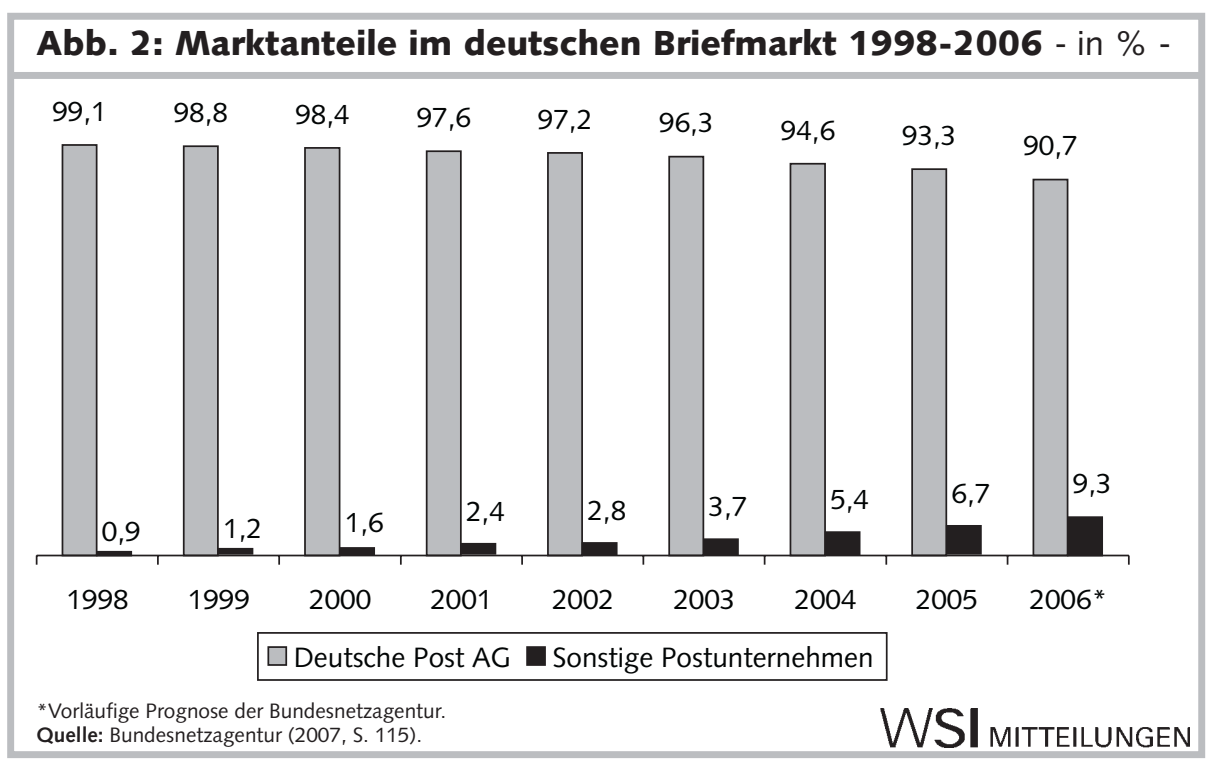


1995 in dem bislang längsten Streik in der Geschichte der Deutschen Post erkämpft werden, in dessen Folge sich die Deutsche Post AG bereit erklärt hat, auf betriebsbedingte Kündigungen zu verzichten (Völlings 2004).

Im Juli 2003 wurde diese Politik in einem „Beschäftigungspakt“ zwischen der Deutsche Post AG und der Vereinten Dienstleistungsgewerkschaft (ver.di) weiter fortgeschrieben, in dem für die Beschäftigten der Unternehmensbereiche Brief und Express betriebsbedingte Kündigungen bis zum 31. März 2008 ausgeschlossen werden. Außerdem verpflichtete sich das Unternehmen, die Fremdvergabe bei der Brief- und Paketzustellung zu begrenzen. Im Jahr 2006 wurden die wesentlichen Regelungen des Beschäftigungspaktes bis zum 31. Dezember 2009 verlängert (Teuscher 2006, S. 7).

Als Gegenleistung für die von der Deutschen Post AG betriebene Politik eines „sozialverträglichen Personalabbaus" waren die Gewerkschaften im Rahmen von Tarifvereinbarungen $\mathrm{zu}$ einigen substanziellen Zugeständnissen bereit, die zu einer deutlich höheren Flexibilität der Arbeitsorganisation und zu teilweise empfindlichen Verschlechterungen bei den Arbeitsbedingungen geführt haben (Völlings 2004; Wehner 2005). Hierzu gehören u.a. die „freiwillige“ Akzeptanz längerer Arbeitszeiten, der Einsatz von mehr Teilzeitkräften, der Verzicht auf arbeitsfreie Tage, die Versetzung von Beschäftigten und die Nutzung von Änderungskündigungen. Außerdem wurden für die neu gegründeten Tochtergesellschaften der Deutschen Post außerhalb der Briefsparte eigene Tarifverträge abgeschlossen, die teilweise deutlich unterhalb der bestehenden Tarifstruktur liegen. ${ }^{5}$

Im Mittelpunkt der tarifvertraglichen Anpassung an die neuen Bedingungen eines liberalisierten Briefmarktes stand hingegen die Entwicklung eines neuen Rahmentarifvertrages, mit dem 2001 zunächst für die Arbeiter und 2003 dann auch für die Angestellten ein neues Entlohnungs- und Eingruppierungssystem eingeführt wurde. Das Ziel dieser Tarifvertragsreform bestand darin, die Entlohnungsstruktur endgültig von dem Vorbild des öffentlichen Dienstes zu lösen und zugleich das Entlohnungsniveau deutlich abzusenken. De facto ist auf diesem Wege eine neue „Zwei-Klassen-Tarifstruktur" entstanden, in der einerseits der Besitzstand der Altbeschäftigten gesichert wurde, während andererseits die
Neueingestellten je nach Lohngruppe bis zu 30 \% Einkommenseinbußen hinnehmen mussten. So verdiente z. B. 2006 ein 35 Jahre alter Fahrradzusteller im fünften Beschäftigungsjahr mit Besitzstandregelung 34.677 Euro. Das Jahresgehalt desgleichen Zustellers ohne Besitzstandregelung lag demgegenüber nur bei 24.580 Euro (DPWN 2006). Gegenwärtig fallen noch etwa drei Viertel aller Beschäftigten unter den Tarifvertrag „DP AG Besitzstand“, während etwa ein Viertel sich mit den niedrigeren Löhnen des „DP AG Tarifvertrag“ zufriedengeben muss, der sich beim Lohnniveau am Tarifvertrag für das Speditionsgewerbe in Nordrhein-Westfalen orientiert.

\subsection{ARBEITSBEZIEHUNGEN UND ARBEITSBEDINGUNGEN BEI DEN NEUEN POSTUNTERNEHMEN}

Im Gegensatz zur Deutschen Post AG existiert bislang in keinem der neu entstandenen Postunternehmen ein Tarifvertrag (Input Consulting 2006b, S. 50). Ähnlich schwach entwickelt sind die betrieblichen Interessenvertretungen. Nach den Ergebnissen einer von Input Consulting im Dezember 2006 vorgelegten Studie gab es lediglich in 3,5\% aller betriebsratsfähigen Postunternehmen einen Betriebsrat (ebd., S. 58). Aufgrund der eher kleinbetrieblichen Struktur der neuen Postunternehmen und der großen Anzahl prekärer Beschäftigungsverhältnisse (befristet Beschäftigte, Minijobber usw.), die eine hohe Fluktuationsrate mit sich bringen, ist es für die Gewerkschaften besonders schwierig, in diesen Bereichen Mitglieder zu gewinnen und betriebliche Interessenvertretungsstrukturen aufzubauen. Hinzu kommt, dass die Bildung von Betriebsräten in einigen Unternehmen teilweise massiv durch die Geschäftsleitung behindert wurde. Insgesamt scheinen die Arbeitsbeziehungen in vielen der neuen Postunternehmen durch eine "autoritär-hierarchische“ Unternehmensund Führungskultur geprägt zu sein: In vielen Unternehmen kann „die von der Unternehmensleitung erwartete Arbeitsleistung ... nach Auffassung des Managements offensichtlich nur durch ständigen ,Druck von oben' sichergestellt werden, vor allem auch deshalb, weil andere Anreizsysteme wie ein leistungsgerechtes Einkommensniveau, ein gesicherter Arbeitsplatz und ein angenehmes Betriebsklima - als Motivationsmittel nicht zur Verfügung stehen" (ebd., S. 59f.)
Angesichts der fehlenden betrieblichen und gewerkschaftlichen Interessenvertretungsstrukturen und tarifvertraglichen Regelungen existieren bei den neuen Postunternehmen im Vergleich zur Deutschen Post AG deutlich schlechtere Lohn- und Arbeitsbedingungen. Nach der Untersuchung von Input Consulting liegen die durchschnittlichen Lohnkosten bei den beiden Hauptkonkurrenten PIN Group und TNT je nach Beschäftigtengruppe zwischen $30 \%$ und $60 \%$ unterhalb der Deutschen Post AG (ebd., S. 90). Die meisten neuen Briefdienstunternehmen zahlen ihren Zustellern einen Stundenlohn zwischen 6,50 und 8 Euro in Westdeutschland und zwischen 5 und 7 Euro in Ostdeutschland (ebd., S. 48).

Nach einer vergleichenden Übersicht von ver.di zahlt die PIN AG in Berlin einem 35 Jahre alten Zusteller einen garantierten Stundenlohn von 5,86 Euro (Tabelle 4). Noch niedriger lag der Stundenlohn mit 5,17 Euro bei der Firma Jurex, die sich insbesondere auf Behördenpost spezialisiert hat. In beiden Unternehmen kann sich der Stundenlohn durch die Zahlung von freiwilligen Prämien theoretisch auf etwas über 8 Euro bei der Pin AG und knapp 7 Euro bei Jurex erhöhen. Demgegenüber liegt der Stundenlohn für einen Zusteller nach dem Tarifvertrag Logistik in Berlin bei 9,47 Euro, während sein Kollege bei der Deutschen Post AG 10,54 Euro pro Stunde erhält. Hinzu kommt, dass der Tarifvertrag der Deutschen Post eine kürzere Wochenarbeitszeit, mehr Urlaubstage und die Zahlung von Urlaubs- und Weihnachtsgeld vorsieht. $^{6}$

\footnotetext{
5 Mit dem in Düsseldorf ansässigen Unternehmen First Mail existiert auch ein im Briefsektor tätiges Tochterunternehmen der Deutschen Post AG, das mit etwa 80 Beschäftigten keiner Tarifbindung unterliegt. Nach Angaben der Deutschen Post AG handelt es sich hierbei allerdings um einen singulären Fall, der als "Testobjekt" dient, „um zu studieren, wie das Geschäftsmodell unserer Konkurrenz funktioniert" (Zumwinkel 2007, S. 194).

6 Im Ausland scheint sich die Deutsche Post AG allerdings ähnlich zu verhalten wie die Konkurrenzunternehmen hierzulande. In den Niederlanden weigert sich z. B. die Deutsche Post-Tochter Selekt Mail bis heute, mit den Gewerkschaften Tarifverhandlungen zu führen. Die Löhne bei Selekt Mail liegen nicht nur weit unter dem Niveau des ehemaligen staatlichen Postkonzerns TNT, sondern durch den Einsatz von auf Stücklohnbasis bezahlten "Selbstständigen" teilweise sogar unterhalb des in den Niederlanden vorgeschriebenen gesetzlichen Mindestlohns (Het Financieele Dagblad vom 21.3.2007).
} 
riger Arbeit gelungen ist, bei der Berliner PIN AG betriebliche Interessenvertretungsstrukturen aufzubauen (Frank 2007). Darüber hinaus hat ver.di gezielt versucht, die Kunden der PIN AG auf die skandalösen Arbeits- und Beschäftigungsbedingungen aufmerksam zu machen. Eine besondere Rolle spielte in diesem Zusammenhang der Berliner Senat, der zu den wichtigsten Großkunden der PIN AG gehört. Letzteres führte zu der paradoxen Situation, dass das Land Berlin zwar einerseits in der Lage war, aufgrund der im Vergleich zur Deutschen Post AG billigeren Tarife der Pin AG Kosten zu sparen. Andererseits haben jedoch zahlreiche PIN-AG Beschäftigte einen Anspruch darauf, dass ihr niedriges Einkommen durch zusätzliche Sozialleistungen nach Hartz IV aufgestockt wird, was wiederum zulasten der kommunalen Haushalte geht. Inzwischen hat sich der Berliner Senat dazu entschieden, zukünftig bei allen öffentlichen Aufträgen eine Tariftreueerklärung zu verlangen. Die für den Sommer 2007 anstehende Vertragsverlängerung mit dem Berliner Senat dürfte deshalb auch den letztendlichen Ausschlag für die PIN Group gegeben haben, mit ver.di in Tarifverhandlungen zu treten.

Neben der PIN Group hat mittlerweile mit TNT auch der zweite große Konkurrent der Deutschen Post AG mit ver.di Vorgespräche über die Aufnahme von Tarifverhandlungen geführt. Beide Unternehmen haben sogar nicht mehr ausgeschlossen, dass es zukünftig einen Flächentarifvertrag für die gesamte Branche geben könnte. Ob es tatsächlich gelingt, das der- zeit äußerst niedrige Lohnniveau bei den privaten Postunternehmen durch Tarifverträge substanziell anzuheben, bleibt jedoch abzuwarten. Die Politik hat es, wie das Beispiel Berlin zeigt, auf jeden Fall in der Hand, diesen Prozess durch die Anwendung von Tariftreueklauseln zu fördern. Schließlich ist es offensichtlich, dass der angedeutete Strategiewechsel bei einigen großen Postunternehmen auch mit der schwindenden gesellschaftlichen und politischen Akzeptanz einer vollständigen Liberalisierung des Briefmarktes zusammenhängt. Für die Politik ließe sich hieraus das Junktim ableiten, dass ohne eine angemessene (tarif-)politische Re-Regulierung des Postsektors keine weiteren Liberalisierungsschritte folgen dürfen.

\section{LITERATUR}

Brandt, T. (2007): Liberalisation, privatisation and regulation of postal servieses in Europe - First international experiences in the run-up to new European regulations, PIQUE working paper, http://www.boeckler.de/ pdf/wsi_pj_piq_post_europe.pdf

Bundesnetzagentur (2006): Neunte Marktuntersuchung für den Bereich der lizenzpflichtigen Postdienstleistungen, Mainz

Bundesnetzagentur (2007): Jahresbericht 2006, Bonn

Cox, H. (1999): Regulierung, Privatisierung und Globalisierung von Post und Telekommunikation. Rückblick und Ausblick über zehn Jahre Postund Telekommunikationsreform in Deutschland, in: Jahrbuch für Wirtschaftsgeschichte "Privates Eigentum - Öffentliches Eigentum“ 1, Berlin, S. $73-100$

Drews, K. (2006): Liberalisation, privatisation and regulation in the German postal services sector, PIQUE country reports on liberalisation and privatisation processes and forms of regulation, Düsseldorf, November [www.boeckler.de/pdf/wsi_pj_piq_sekpost.pdf]

Deutsche Post World Net (2006): Vergleich der Beschäftigungsbedingungen - Analyse Jahresgesamtentgelt, Chart aus der Tarifrunde 2006, Manuskript

Deutsche Post World Net (2007a): Geschäftsbericht 2006, Bonn Deutsche Post World Net (2007b): Klarstellungen zum Jahresbericht der Bundesnetzagentur, Manuskript Europäische Kommission (2006): Vorschlag für eine Richtlinie des Europäischen Parlamentes und des Rates zur Änderung der Richtlinie 97/67/EG über die Vollendung des Binnenmarktes für Postdienste. Brüssel, 18.10., KOM(2006) 594 endgültig
Frank, B. (2007): PIN-AG: Hat die unendliche Geschichte jetzt ein Ende?, in: betrieb \& gewerkschaft Intern 1, Berlin, S. 7-8

Haas, R./Auer, H./Keseric, N./Stefanescu, G. (2004): Liberalisierung öffentlicher Dienstleistungen in der Europäischen Union und Österreich. Auswirkungen auf Preise, Qualität, Versorgungssicherheit und Universaldienste in den Sektoren Energie und Post, Arbeiterkammer Wien, Studien zur Zukunft öffentlicher Dienstleistungen Band 3, Wien

Input Consulting (2006a): Im Gleichklang? Befunde zur Liberalisierung und Regulierung des Postsektors in ausgewählten EU-Mitgliedstaaten, Stuttgart

Input Consulting (2006b): Liberalisierung und Prekarisierung - Beschäftigungsbedingungen bei den neuen Briefdienstleistern in Deutschland, Dezember, Stuttgart

Lippert, I. (2005): Öffentliche Dienstleistungen unter EU-Einfluss. Liberalisierung - Privatisierung - Restrukturierung - Regulierung, Berlin Lotz, W. (2007): Die deutsche Post von der Postreform I bis zum Börsengang 1989-2000, Frankfurt a. M.

Pin Group/ver.di (2007): Vereinbarung 29. Januar, Berlin Postregulationsbehörde (2007): Dokumentation: Einhaltung der branchenüblichen Arbeitsbedingungen als Konzessionsvoraussetzung, 8. Januar, Bern

Raza, W./Wedl, V./Angelo, S. (2004): Liberalisierung öffentlicher Dienstleistungen. Eine konzeptuelle, begriffliche und rechtliche Einführung ins Thema, Arbeiterkammer Wien, Studien zur Zukunft öffentlicher Dienstleistungen Band 1, Wien 
Säcker, F. (2007): Soziale Schutzstandards im Postregulierungsrecht. Rechtsgutachten für die Bundesnetzagentur für Elektrizität, Post, und Eisenbahn, Januar, Berlin

Scheurle, W. (2001): Vom Sondervermögen des Bundes zum Konzern Deutsche Post World Net, Beitrag für das Symposium zur Verwaltungsmodernisierung am 6./7. 12. 2001 in Herne [www.im.nrw.de/inn/seiten/ vm/herne_01/vortraege/scheuerle.pdf]

Teuscher, F. (2006): Schutz vor Fremdvergabe in der Paketzustellung verlängert, in: bewegen 12, S. 7

ver.di (2007): Entwicklungen im Bereich „Neue Briefdienstleister“, Bundesfachgruppenvorstand Postdienste, 5./6. Februar, Manuskript
Völlings, H. (2004): Die Privatisierung und Deregulierung des Postsektors, in: Lorenz, F./Schneider, G. (Hrsg.): Wenn öffentliche Dienste privatisiert werden, Hamburg, S. 76-80

Wehner, E. (2005): Von der Bundespost zu den Global Players Post AG + Telekom AG. Profiteure und Verlierer der Privatisierung, Institut für sozialökologische Wirtschaftsforschung, ISW-Report 64, München

Zumwinkel, K (2007): „Das kostet 32.000 Jobs“, Interview mit Klaus Zumwinkel, in: Stern Nr. 13 vom 22.3., S. 192-195

\title{
Call for Papers
}

Der SAMF plant in Kooperation mit dem WSI und dem SFB 580 seine Jahrestagung 2007 zum Thema

\section{Arbeitsmarkt und Sozialpolitik Kontroversen um Effizienz und Sicherheit}

\author{
13. und 14. Dezember 2007 in Berlin
}

Arbeitsmärkte sind durch eine Zunahme an Flexibilität und Mobilität gekennzeichnet. Inwieweit sich hieraus Chancen und Risiken ergeben, ist u.a. von der Ausgestaltung der sozial- und arbeitsmarktpolitischen Gestaltung abhängig. Ziel der Tagung ist es, die sozial- und arbeitsmarktpolitischen Funktionsvoraussetzungen für flexible Arbeitsmärkte näher zu bestimmen, die sowohl Effizienzals auch Sicherheitskriterien entsprechen.

Vortragsvorschläge sind für folgende zwei Themenfelder willkommen:

Wechselwirkungen zwischen Arbeitsmärkten und Sozialpolitik

Die Beiträge sollen analysieren: Welche Anforderungen an Qualifizierung, soziale Sicherung und Leistungsbereitschaft stellen flexible Arbeitsmärkte? In welcher Weise tragen die derzeitige Sozial- und Arbeitsmarktpolitik des Staates sowie jene von Unternehmen oder Tarifparteien dazu bei, soziale und ökonomische Risiken sowie Investitions- und Planungsunsicherheit zu fördern oder zu vermindern? Welche Personengruppen sind positiv oder negativ betroffen? Inwieweit sind zentrale Bereiche der gesellschaftlichen Reproduktion wie Bildung, Engagement, Fertilität etc. durch zunehmende Flexibilität und Mobilität auf Arbeitsmärkten gefährdet?

Effizienz und Sicherheit: Sozialpolitische Wege in Europa

In Europa werden unterschiedliche Wege beschritten, um Effizienz und soziale Sicherheit auf Arbeitsmärkten zu steigern. In den Beiträgen sollen die Erfahrungen mit unterschiedlichen institutionellen Regelungsformen des Arbeitsmarktes und mehr oder minder kommodifizierenden und segmentierenden sozialen Sicherungssystemen vorgestellt werden. So sollen sie Antwort geben auf die Frage: Welche Erfahrungen lassen sich im positiven oder negativen Sinne als beispielgebend für Deutschland kennzeichnen?

Abstracts mit max. 2.500 Zeichen sollen bis zum 22. Juli 2007 in elektronischer Form (vorzugsweise als pdf-Datei) an Helga-Faasch@boeckler.de geschickt werden. Die Benachrichtigung über die Annahme eines Beitrags erfolgt bis zum 15. August 2007.

Juroren und Organisatoren sind:

PD Dr. Matthias Knuth (Institut für Arbeit und Qualifikation, Universität Duisburg/ Essen);

Prof. Dr. Bernd Reissert (Hochschule der Bundesagentur für Arbeit, Mannheim);

Dr. Hartmut Seifert (WSI in der Hans-Böckler-Stiftung, Düsseldorf);

Dr. Barbara Skripski (Senatsverwaltung für Gesundheit, Soziales und Verbraucherschutz, Berlin);

PD Dr. Olaf Struck (Inst. f. Soziologie, Friedrich-Schiller-Universität Jena und SFB 580). 